\title{
The Influence of Recycling on the Properties of Interface between Ceramic and Dental Alloys
}

\author{
Yinghui Wang $\mathbb{D}^{1}$, Honglan Huang, ${ }^{2,3}$ Honglei Lin, ${ }^{2}$ Lei Jiang, ${ }^{2}$ Yu Pan, ${ }^{2}$ Xiurong Li, \\ and Hui Cheng $\mathbb{B}^{2}$ \\ ${ }^{1}$ Fujian Key Laboratory of Oral Diseases \& Fujian Provincial Engineering Research Center of Oral Biomaterial \& Stomatological Key \\ lab of Fujian College and University, School and Hospital of Stomatology, Fujian Medical University, Fuzhou, Fujian, China \\ ${ }^{2}$ Institute of Stomatology \& Research Center of Dental Esthetics and Biomechanics, School and Hospital of Stomatology, \\ Fujian Medical University, Fuzhou, Fujian, China \\ ${ }^{3}$ Stomatological Hospital of Xiamen Medical College, Xiamen, Fujian, China \\ ${ }^{4}$ Department of Prosthetic Technology, School and Hospital of Stomatology, Fujian Medical University, Fuzhou, Fujian, China
}

Correspondence should be addressed to Hui Cheng; huicheng.fjmu@yahoo.com

Received 29 December 2019; Accepted 16 March 2020; Published 8 April 2020

Academic Editor: Adriana Bigi

Copyright (C) 2020 Yinghui Wang et al. This is an open access article distributed under the Creative Commons Attribution License, which permits unrestricted use, distribution, and reproduction in any medium, provided the original work is properly cited.

\begin{abstract}
Statement of Problem. Results are discrepant regarding the metal-ceramic interface of dental alloys affected by recycling. Purpose. The purpose of this study was to evaluate the effect of recycling on the properties of interface between 2 dental alloys and their corresponding porcelains. Materials and Methods. Noble alloy (Pd-Cu-Ga) and high-noble alloy (Au-Pt) were used in this study. Metal matrices (cylinders $\Phi 4 \mathrm{~mm} \times 4 \mathrm{~mm}$ with pedestal $\Phi 5 \mathrm{~mm} \times 1 \mathrm{~mm}$ ) were prepared by arc melting in argon after recasting 1-3 times. Corresponding porcelain with overall dimensions of $\Phi 4 \mathrm{~mm} \times 2 \mathrm{~mm}$ was veneered on each metal cylinder. There were 22 specimens in each alloy group. Specifically, two specimens of each group were chosen randomly for interfacial morphology and diffusion analyses by scanning electron microscopy (SEM) equipped with energy-dispersive X-ray spectroscopy (EDS). The remaining 20 specimens were divided into 2 groups with or without thermal cycling. The bond strength was evaluated by shear test, and the data were analyzed by two-way analysis of variance (ANOVA). The failure mode of shear test specimen was observed with a stereoscopic microscopy and subjected to the exact probability test $(\alpha=0.05)$. Results. According to the results from SEM, no obvious difference was observed in the interfacial morphology of both $\mathrm{Pd}-\mathrm{Cu}-\mathrm{Ga}$ and $\mathrm{Au}-\mathrm{Pt}$ alloys among different recasting specimens. EDS analysis revealed that no significant difference was found in the width of elemental diffusion among 2 test alloys after recycling 1-3 times. Notably, in Pd-Cu-Ga alloy groups, the peak of Ga in thrice recasting was lower than those in first and second recastings. And there was no significant difference $(P>0.05)$ in the metal-ceramic shear bond strength of Pd-Cu-Ga and Au-Pt alloys after recycling 1-3 times, with or without thermal cycling. The results of failure modes observed on specimens were not affected by the recycling and thermal cycling in the 2 tested alloys. Conclusions. Within the limitations of this study, the $\mathrm{Pd}-\mathrm{Cu}-\mathrm{Ga}$ and $\mathrm{Au}-\mathrm{Pt}$ alloys can be recycled 2 times without significant changes on the properties of metal-ceramic interface, with or without thermal cycling.
\end{abstract}

\section{Introduction}

As all-ceramic restorations, CAD/CAM and 3-dimensional printing technology are now available in dental clinics; conventional casting metal-ceramic restorations are not the only choice for dentists and patients. However, compared to metal-ceramic restorations, the long-term outcomes and complications of these new restorations are still not well understood. For example, the incidence rate of catastrophic framework fracture and veneer chipping in all-ceramic restorations is higher than similar metal ceramic restorations $[1,2]$, and there is still no CAD/CAM and 3-dimensional printing technology with noble and high-noble alloys. Therefore, conventional casting of noble and high-noble alloys 
TABLE 1: Compositions of 4 ceramic alloys as provided by manufacturer (wt\%).

\begin{tabular}{lcc}
\hline Alloy types & Manufacturer & Composition (wt\%) \\
\hline Pd-Cu-Ga & Albabond E, HeraeusKulzer, Wertheim, Germany & Pd: 78.0, Cu: 10.8, Ga: 7.5, Au: 1.6, In: 1.3, Ru: 0.4, Zn: 0.2, Sn: 0.2 \\
Au-Pt & Alfa Ceramic 90, Alldental, Stockholm, Sweden & Au: 89.5, Pt: 5.8, Pd: 1.6, Ag: 1.2, Ir: 0.6, Sn: 0.3, In: 0.8, Fe: 0.2 \\
\hline
\end{tabular}

TABle 2: Test specimen groups.

\begin{tabular}{lcccc}
\hline Alloy type & Thermal cycling & Cast once & Cast twice & Cast thrice \\
\hline \multirow{2}{*}{ Pd-Cu-Ga } & Yes & Pd-1t & Pd-2t & Pd-3t \\
& No & Pd-1 & Pd-2 & Pd-3 \\
\hline \multirow{2}{*}{ Au-Pt } & Yes & Au-1t & Au-2t & Au-3t \\
& No & Au-1 & Au-2 & Au-3 \\
\hline
\end{tabular}

metal-ceramic restorations will still likely remain as one of the proven restorative options for patients in the near future.

However, the conventional lost-wax metal casting process of metal-ceramic restorations usually produces a large amount of dental alloy waste, such as the sprues and cast investments, thus increasing medical costs, wasting metal resource, and polluting the environment as well. So nowadays, noble and high-noble alloys are commonly recycled in dental laboratories.

A number of authors investigated the feasibility of recycling dental alloys, but these studies varied in research methodology and designs. There was no agreeable test and evaluation protocol for recycling yet. [3] Our recent study has established a series of mechanical and chemical treatments for previously melted buttons or sprues before recycling, and the results showed that the contamination of previously cast ceramic alloys could be effectively removed. [4] After recasting thrice without adding any new alloys by electric arc melting under an argon gas-protective vacuum environment, we found that there was no significant decline of tensile strength, $0.2 \%$ yield strength, flexural strength, flexural modulus, and Vickers hardness in $\mathrm{Co}-\mathrm{Cr}$ and $\mathrm{Au}-$ Pt alloys but decrease of tensile properties in cpTi [5-7].

In addition to the mechanical properties, the metalceramic compatibility is also of great importance to the clinical success. Hence, the purpose of this study was to evaluate the effect of recasting on the properties of interface between 2 dental alloys (noble and high-noble) and their corresponding porcelains after recasting 1-3 times.

\section{Materials and Methods}

The present study tested noble alloy (Pd-Cu-Ga) and highnoble alloy (Au-Pt) after recasting 1-3 times, with or without thermal cycling. Manufacturers and composition of the ceramic alloys used are shown in Table 1.

2.1. Specimens Preparation. 132 wax specimens (cylinder $\Phi$ $4 \mathrm{~mm} \times 4 \mathrm{~mm}$ with a pedestal $\Phi 5 \mathrm{~mm} \times 1 \mathrm{~mm}$ ) were randomly and equally divided into 6 groups $(n=22)$ as shown in Table 2. The wax specimens were sprued and invested with a phosphate-based investment (Vesto-Fix, DFS, Germany)
TABLE 3: Firing procedures of the dental ceramics.

\begin{tabular}{lccccc}
\hline Ceramics & $\begin{array}{c}\text { ST } \\
\left({ }^{\circ} \mathrm{C}\right)\end{array}$ & $\begin{array}{c}\mathrm{DT} \\
(\mathrm{min})\end{array}$ & $\begin{array}{c}\text { TRI } \\
\left({ }^{\circ} \mathrm{C} / \mathrm{min}\right)\end{array}$ & $\begin{array}{c}\mathrm{FT} \\
\left({ }^{\circ} \mathrm{C}\right)\end{array}$ & $\begin{array}{c}\mathrm{HT} \\
(\mathrm{min})\end{array}$ \\
\hline VMK95 & & & & & \\
$\quad$ Oxidation & 500 & 6 & 55 & 930 & 1 \\
First opaque layer & 500 & 2 & 80 & 950 & 1 \\
Second opaque layer & 500 & 6 & 55 & 930 & 1 \\
First dentin layer & 500 & 6 & 55 & 930 & 1 \\
Second dentin layer & 500 & 6 & 55 & 920 & 1 \\
Glaze firing & 500 & 2 & 55 & 915 & 1 \\
Duceratin & & & & & \\
$\quad$ Bonder & 600 & 6 & 100 & 750 & 1 \\
First opaque layer & 450 & 10 & 100 & 760 & 0.5 \\
Second opaque layer & 450 & 10 & 100 & 760 & 0.5 \\
First dentin layer & 450 & 9 & 55 & 760 & 1 \\
Second dentin layer & 450 & 8 & 55 & 750 & 1 \\
Glaze firing & 450 & 6 & 55 & 730 & 1 \\
\hline
\end{tabular}

ST: starting temperature; DT: drying time; TRI: temperature rate of increase; FT: final temperature; HT: holding time.

according to the manufacturer's instructions. Then 2 ceramic alloys were melted in individual crucibles under an argon gas-protective vacuum environment. After the castings had been cooled at room temperature, they were airborneparticle abraded with $120 \mu \mathrm{m} \mathrm{Al}_{2} \mathrm{O}_{3}$ and cleaned in distilled water to remove residual investment on the surface. Next, alloy specimens were separated from the castings. The buttons and sprues from the first and second castings were prepared according to the treatment protocols that we have previously tested and published as the following [4]: (1) $\mathrm{Pd}-\mathrm{Cu}-\mathrm{Ga}$ ceramic alloys: glass bead airborne-particle abrasion and 30 mins immersion in $40 \%$ HF solution; (2) Au-Pt ceramic alloys: glass bead airborne-particle abrasion. No new alloys were added for the second or third castings for all groups.

The surfaces of specimens were polished with silicon carbide abrasive paper from 240 grit to 600 grit. Then the surface of each alloy was abraded by airborne particles as the following previously established protocols [4]: (1) Pd-Cu-Ga ceramic alloys: $120 \mu \mathrm{m} \mathrm{Al}_{2} \mathrm{O}_{3}$ at an angle of $45^{\circ}$ for $15 \mathrm{~s}$ from a distance of approximately $2 \mathrm{~cm}$, under $0.2 \mathrm{MPa}$; (2) Au-Pt ceramic alloys: $180 \mu \mathrm{m}$ glass bead at an angle of $45^{\circ}$ for $15 \mathrm{~s}$ from a distance of approximately $2 \mathrm{~cm}$, under $0.2 \mathrm{MPa}$, and then ultrasonic cleaned in isopropyl alcohol and distilled water for 5 mins and drying at room temperature. Notably, the surface of $\mathrm{Pd}-\mathrm{Cu}-\mathrm{Ga}$ specimens were oxidized before airborne-particle abrasion. All the specimens were veneered using a costumed mold, 


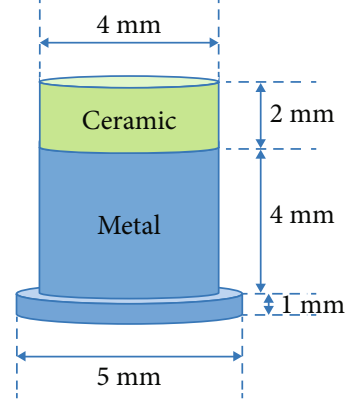

(a)

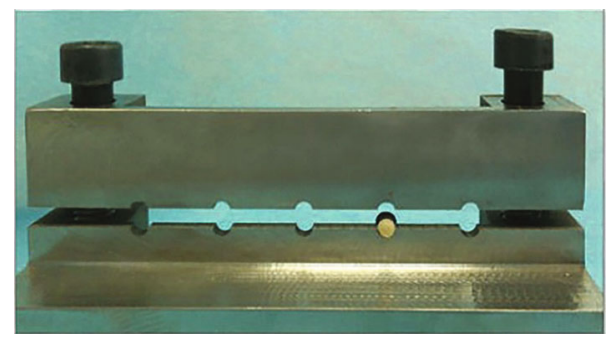

(c)

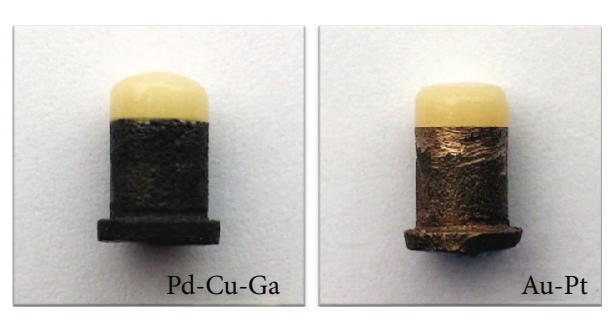

(b)

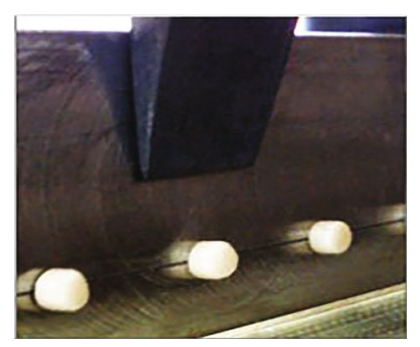

(d)

FIgURE 1: (a) Specimens of 2 tested alloys. (b) Dimensions of the ceramic-alloy specimens. (c) The holder of specimens for shear bond test. (d) The loading device of shear bond test.

including two layers of opaque and body porcelain, respectively, following with glazing as the last step (Table 3 and Figures $1(\mathrm{a})$ and $1(\mathrm{~b}))$. The porcelain powder was VMK95 (VMK 95, Vita, Germany).

More specifically, 2 specimens of each tested alloy were chosen randomly for SEM observation of interfacial morphology and diffusion analysis. The remaining 20 specimens were divided into 2 groups: one with and the other without thermal cycling. The groups without thermal cycling were stored in distilled water for $24 \mathrm{~h}$ at $37^{\circ} \mathrm{C}$, while the groups with thermal cycling were subjected to 3,000 thermal cycles (between 5 and $55^{\circ} \mathrm{C}$, dwell time: $30 \mathrm{~s}$, transfer time: $10 \mathrm{~s}$ ).

2.2. Interfacial Morphology and Diffusion Analysis. According to the method of metallographic microscopic structure examination (GB-T13298-2015) [8], two specimens chosen randomly from each group were embedded in autopolymerizing acrylic resin, and then the lateral surface of the specimens was abraded with silicon carbide abrasive paper from 240 grit to 1,500 grit in order to expose the metal-ceramic interface. They were subsequently polished with diamond paste in the buffing machine with water coolant.

The interfaces of $\mathrm{Pd}-\mathrm{Cu}-\mathrm{Ga}$ specimens were gold-sprayed within an ion sputtering equipment, and then interface diffusion and morphology were assessed by SEM (SU-70, HITACHI, Japan) equipped with EDS analysis (Oxford, X-Max, Great Britain). Considering the influence of gold ions on interface diffusion analysis in Au-Pt specimens, interface diffusion analysis of them was conducted before gold spraying.

2.3. Shear Bond Strength Test. Specimens were subjected to the shear test at room temperature in a universal material testing machine (Model 1342, INSTRON, UK). The load was applied to the metal-ceramic interface at a constant speed of $0.5 \mathrm{~mm} / \mathrm{min}$, and the load on the test specimen was recorded at failure. (Figures 1(c) and 1(d)).

Shear bond strength was analyzed by two-way analysis of variance (ANOVA) and followed with Tukey honest significant difference (HSD) test. The data were analyzed by the Statistical Package Social Sciences (version 21) software (SPSS, Chicago, IL, USA), and $P$ values less than 0.05 were considered statistically significant.

2.4. Failure Type Observation. Failure type ((1) cohesive failure, (2) adhesive failure, (3) mixed failure) of each specimen was determined by the stereoscopic microscopy (ZSA302, COIC, China).

Exact probability test was used to analyze whether recasting has an impact on the metal-ceramic failure type $(\alpha=0.05)$.

\section{Results}

3.1. Interfacial Morphology and Diffusion Analysis. As shown in Figure 2, three different regions of specimens were measured, metal matrix $(\mathrm{M})$, porcelain layer $(\mathrm{P})$, and interfacial layer, according to the cross-sectional morphology under SEM. The results indicated that the metal-ceramic interfaces combined closely in most tested alloys without pores and cracks, indicating good wettability and an appropriate adhesion. Generally, no obvious difference was observed in the interfacial morphology of all tested alloys among different recasting times.

EDS analysis at the interface of all tested alloys after recasting 1-3 times revealed elemental diffusion across the metal-ceramic interface (Figures 3 and 4). In general, no significant difference was found in the width of elemental 


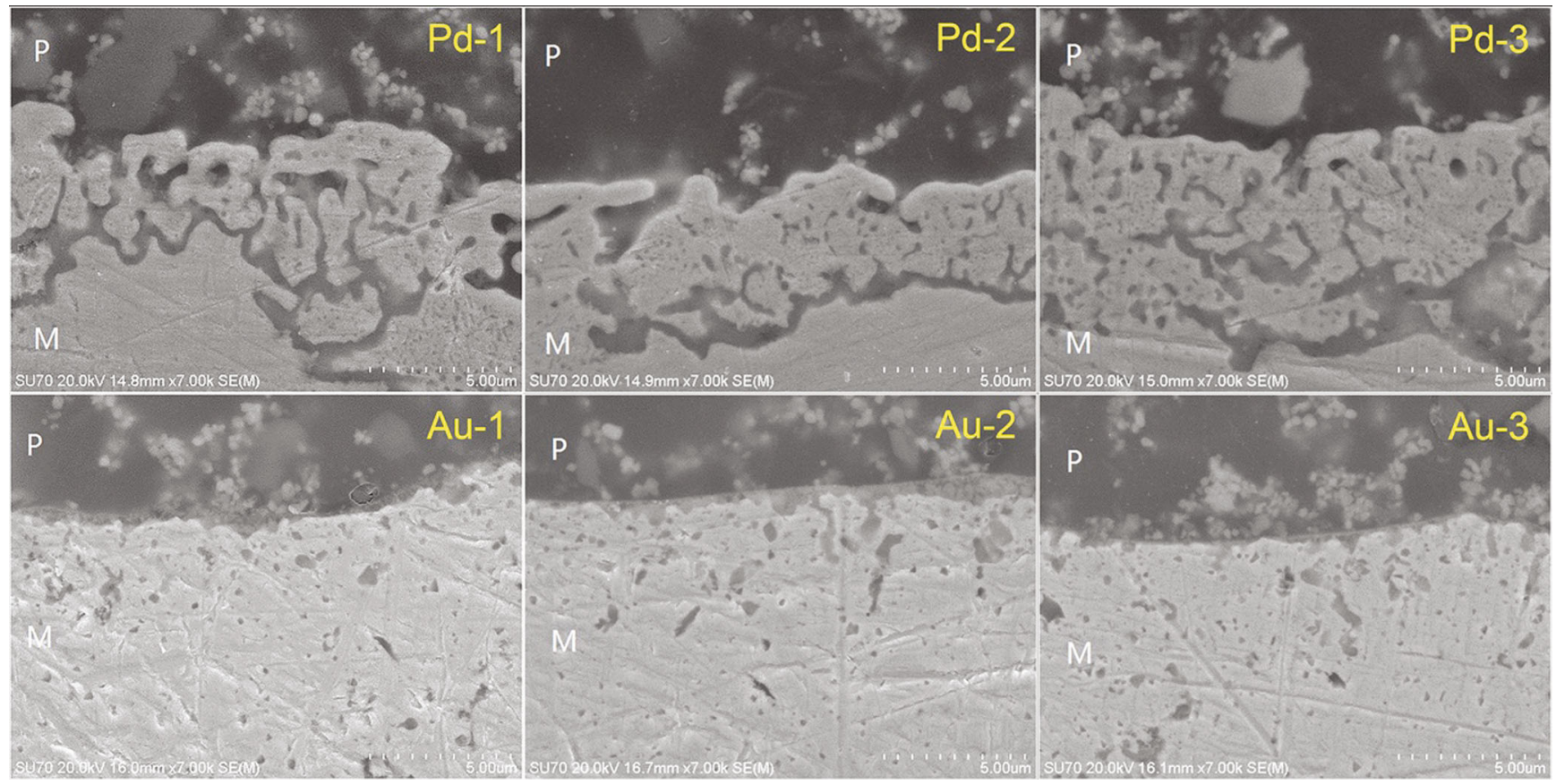

Figure 2: Morphology of metal-ceramic interface of Pd-Cu-Ga and Au-Pt $(\times 7,000)$ alloys after recasting 1-3 times under SEM.
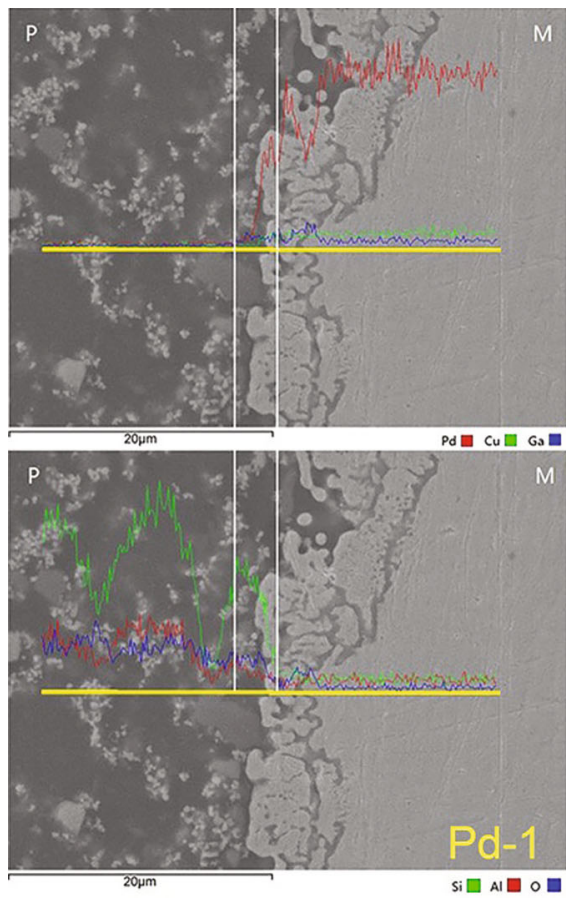
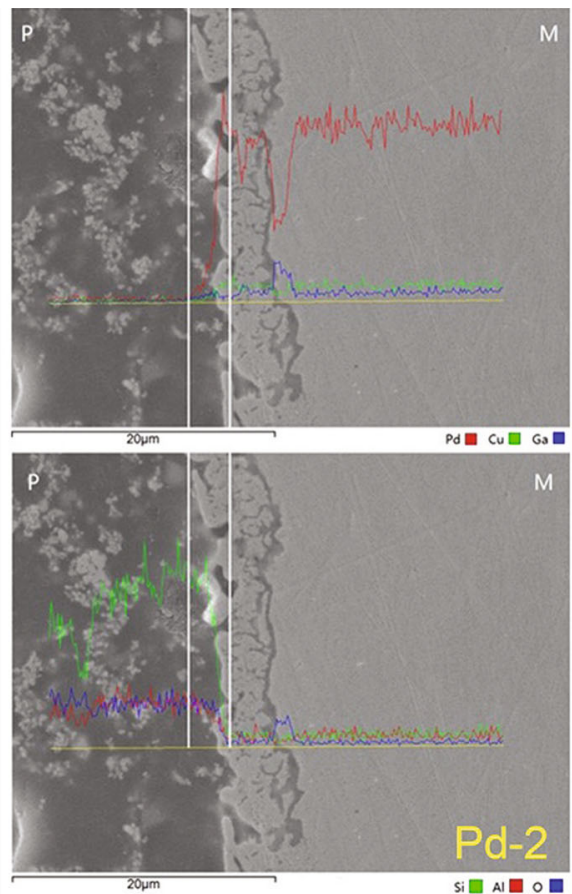
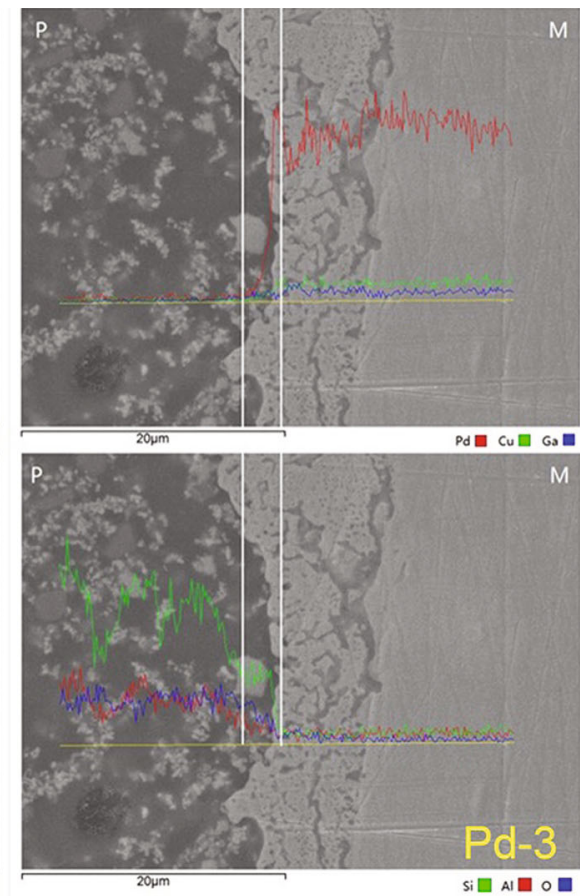

FIgURE 3: Metal-ceramic interfacial diffusion analysis of Pd-Cu-Ga alloy after recasting 1-3 times by SEM equipped with an EDS ( $\times 3,000)$.

diffusion on 2 test alloys after recasting 1-3 times. Notably, in $\mathrm{Pd}-\mathrm{Cu}-\mathrm{Ga}$ alloy, the peak of $\mathrm{Ga}$ in thrice recasting was lower than those in first and second recastings.

3.2. Shear Bond Strength. As shown in Figure 5, there was no significant interaction between recasting and thermal cycling $\quad\left(F_{\mathrm{Pd}-\mathrm{Cu}-\mathrm{Ga}}=0.007, \quad P_{\mathrm{Pd}-\mathrm{Cu}-\mathrm{Ga}}=0.993 ; \quad F_{\mathrm{Au}-\mathrm{Pt}}=\right.$
0.074, $P_{\mathrm{Au}-\mathrm{Pt}}=0.929$ ), and no significant difference in the metal-ceramic shear bond strength of $\mathrm{Pd}-\mathrm{Cu}-\mathrm{Ga}$ and $\mathrm{Au}-$ Pt alloys after recasting 1-3 times, whether subjected to the thermal cycling or not (Recasting: $F_{\mathrm{Pd}-\mathrm{Cu}-\mathrm{Ga}}=0.177$, $P_{\mathrm{Pd}-\mathrm{Cu}-\mathrm{Ga}}=0.838 ; F_{\mathrm{Au}-\mathrm{Pt}}=0.403, P_{\mathrm{Au}-\mathrm{Pt}}=0.912 ;$ Thermal cycling: $\quad F_{\mathrm{Pd}-\mathrm{Cu}-\mathrm{Ga}}=1.598, \quad P_{\mathrm{Pd}-\mathrm{Cu}-\mathrm{Ga}}=0.212 ; \quad F_{\mathrm{Au}-\mathrm{Pt}}=$ $\left.0.538, P_{\mathrm{Au}-\mathrm{Pt}}=0.466\right)$. 

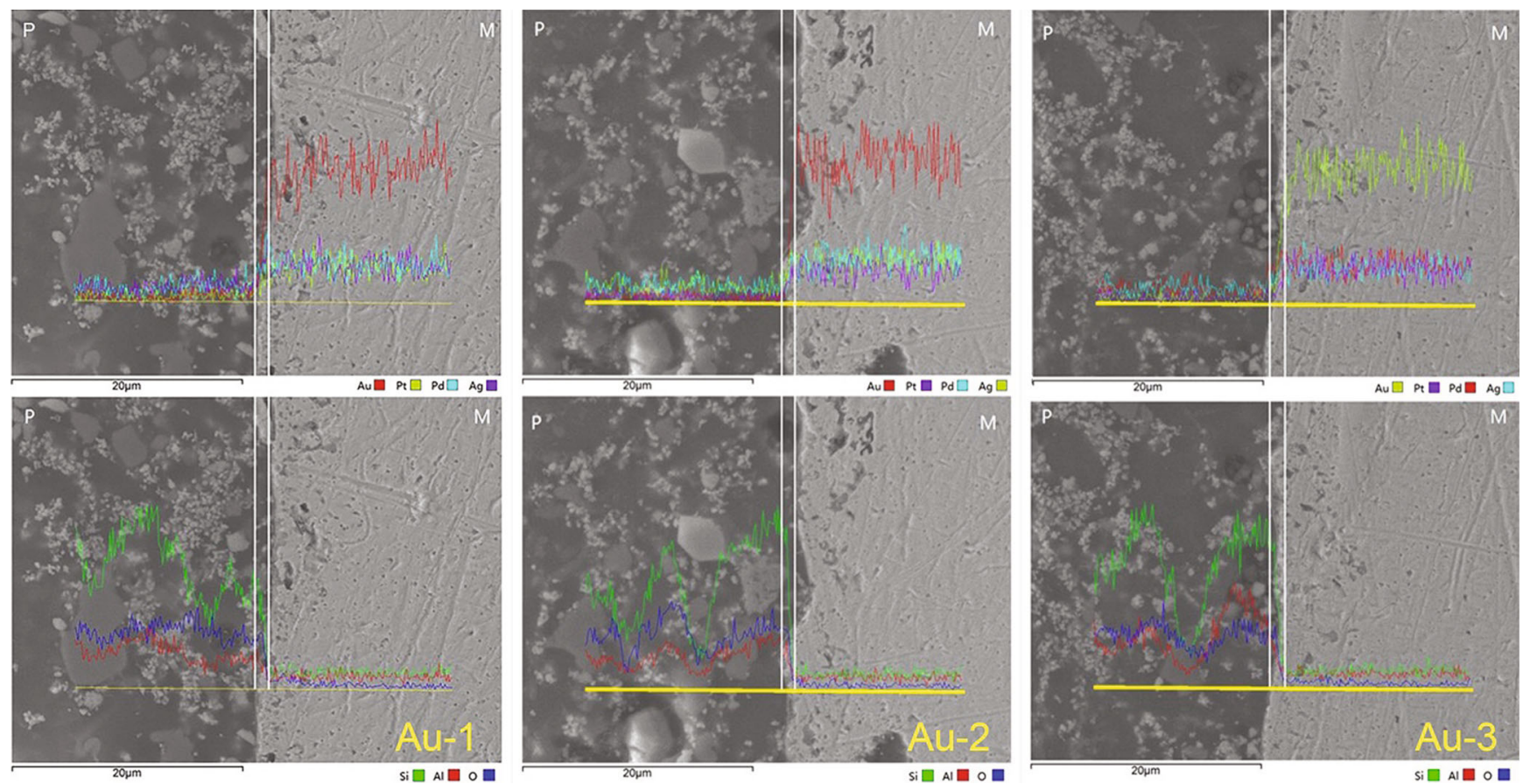

FIGURe 4: Metal-ceramic interfacial diffusion analysis of Au-Pt alloy after recasting 1-3 times by SEM equipped with an EDS $(\times 3,000)$.
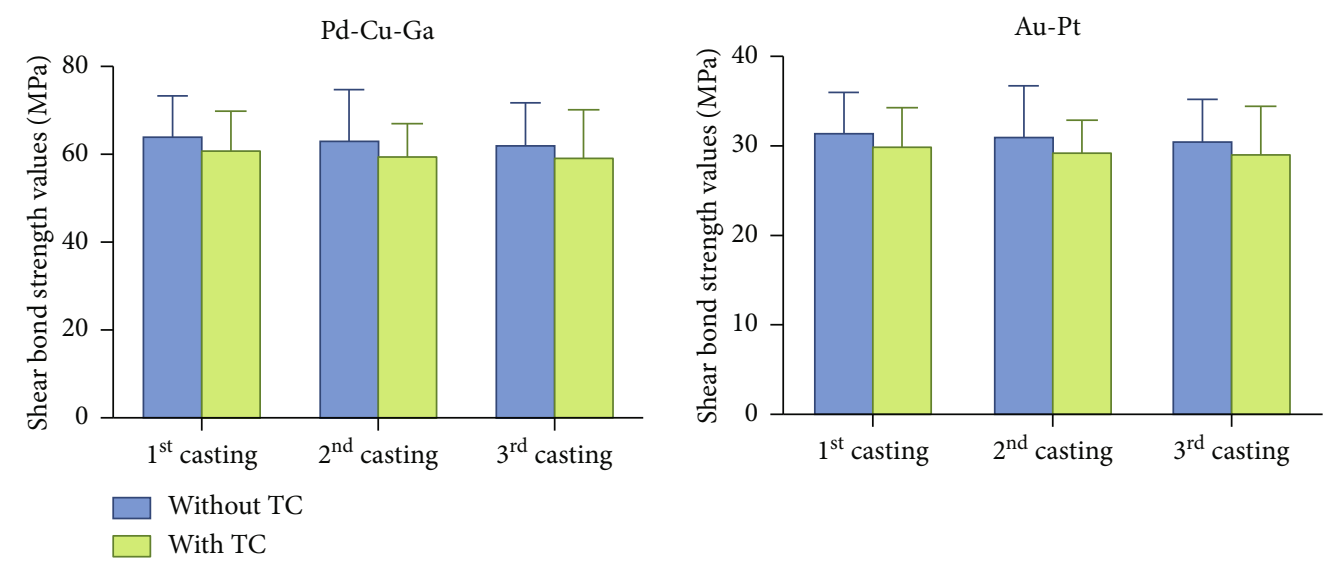

FIGURE 5: Shear bond strength of 2 tested alloys after recasting 1-3 times with and without thermal cycling.

3.3. Failure Type Observation. The fracture types were classified according to the presence or not of ceramic remnants on the metal substrate after shear tests. It was described as follows: (1) adhesive, if no remnants of ceramic were found on the metal surface; (2) cohesive, if fracture occurred within the ceramic side; and (3) mixed, if remnants of ceramic were found in the metal surface. In this study, there was a few cohesive fracture modes in the $\mathrm{Au}-\mathrm{Pt}$ group (Figure 6), and only mixed mode was found in $\mathrm{Pd}-\mathrm{Cu}-\mathrm{Ga}$ group. (Figure 7).

According to the exact probability analysis, there was no significant difference $(P>0.05)$ in the metal-ceramic restorations failure type of all tested alloys after recasting 1-3 times, whether subjected to the thermal cycling or not. (Figure 8).

\section{Discussion}

In this study, a favorable metal-ceramic combination of Pd$\mathrm{Cu}-\mathrm{Ga}$ and $\mathrm{Au}-\mathrm{Pt}$ alloy-ceramic restorations was observed, and the width of the oxide layer analyzed by elemental diffusion did not change significantly on 2 ceramic alloys after recasting 1-3 times. These indicated that recasting 2 times had no obvious negative effects on the oxidation zone of metal-ceramic. It is known that the metal-ceramic bonding is fundamentally based on micromechanical retention, compressive adaptation, chemical union, and Van der Waals forces [9]. The oxide zone is important for forming chemical combination between metal and porcelain, and its thickness directly affects the metal porcelain bond strength. If the oxide layer is thick or loose, its thermal expansion coefficient will 

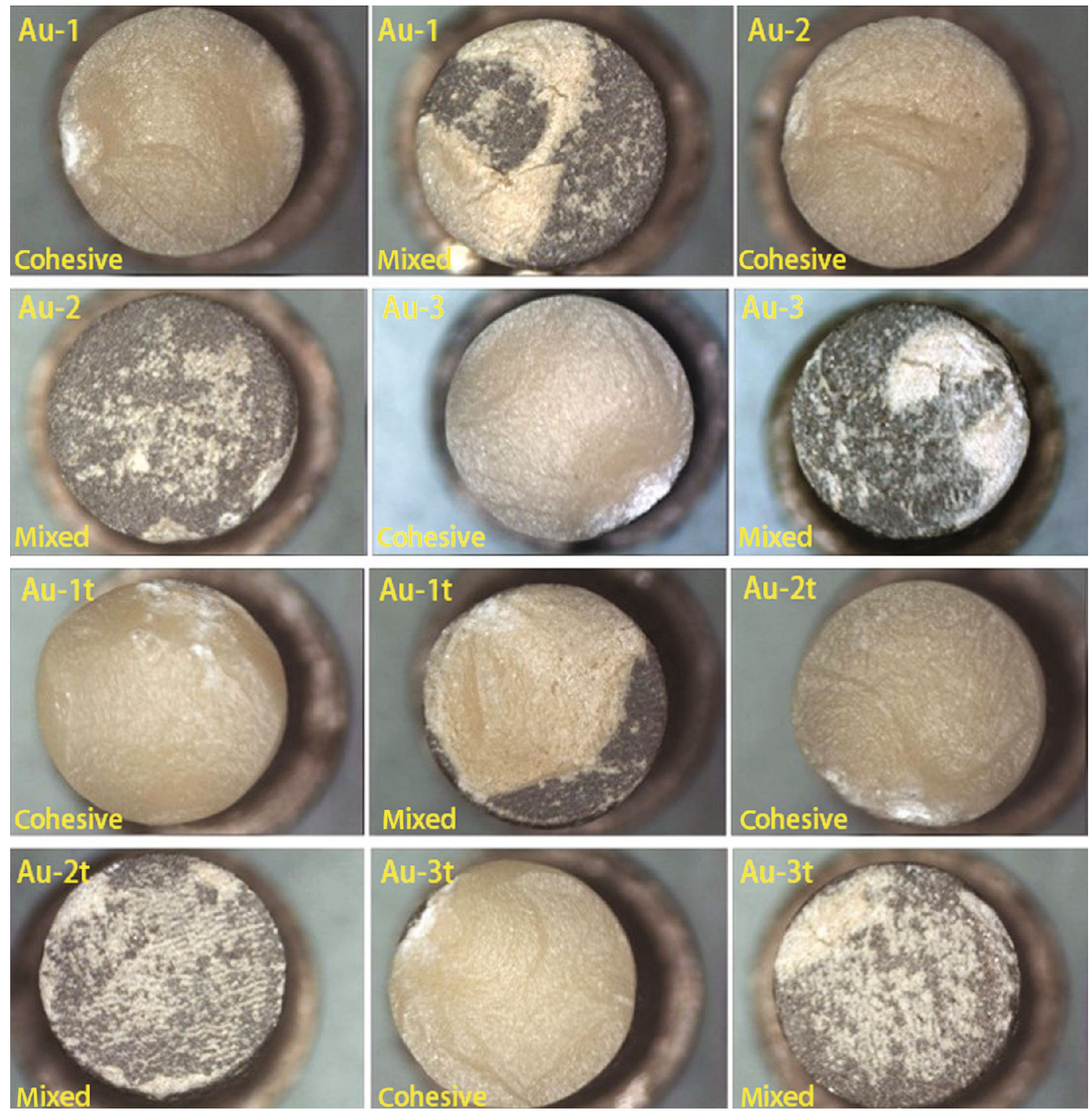

FIgURE 6: Failure type of Au-Pt alloy after recasting 1-3 times with or without thermal cycling.
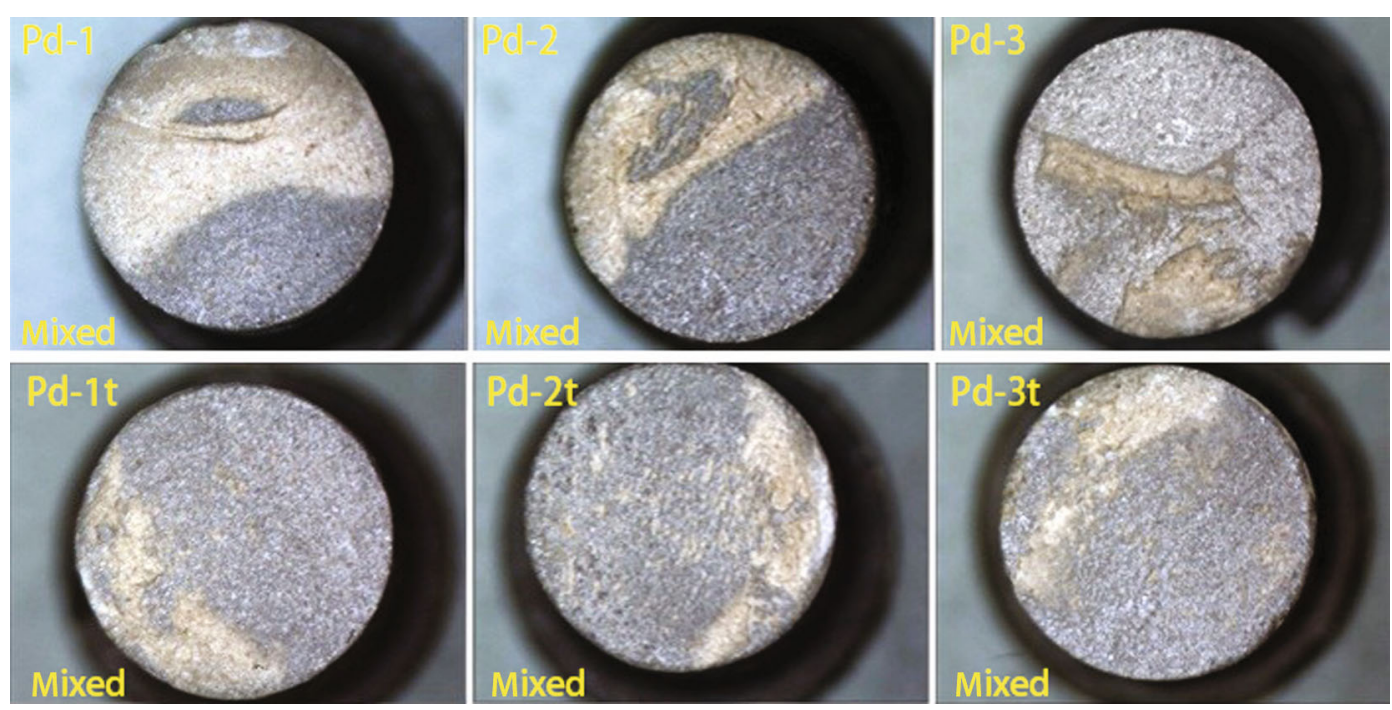

FIGURe 7: Failure type of Pd-Cu-Ga alloy after recasting 1-3 times with or without thermal cycling. 
Pd-Cu-Ga

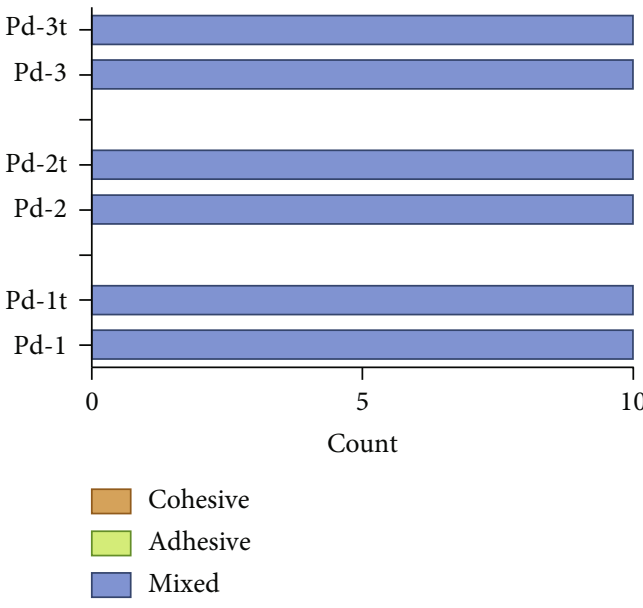

$\mathrm{Au}-\mathrm{Pt}$

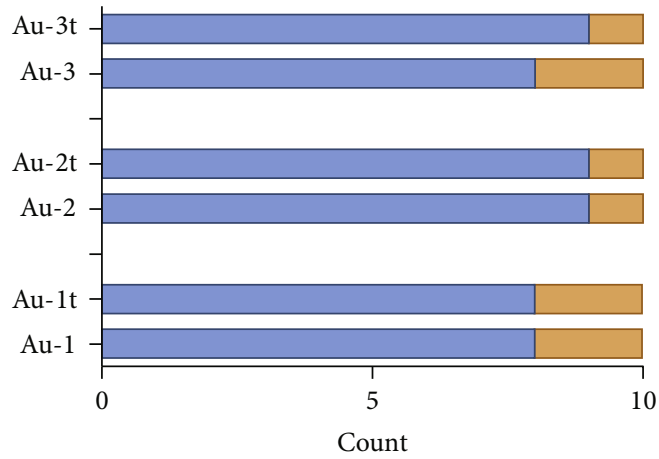

Figure 8: Failure type of 2 tested alloys after recasting 1-3 times with or without thermal cycling.

mismatch with the ones of metal and porcelain, resulting in residual stress in the interface and decreased bond strength. On the other hand, if the oxide layer is too thin, it will be completely soluble in porcelain in the sintering process, making direct contact of porcelain with the metal base by a van der Waals force rather than the chemical bonding, and this also reduces the metal-ceramic bond strength.

The results of shear bond strength and failure type further verified the results of interfacial morphology and diffusion analysis. No significant difference was found in the metal-ceramic bond strength of $\mathrm{Pd}-\mathrm{Cu}-\mathrm{Ga}$ and $\mathrm{Au}-\mathrm{Pt}$ alloys after recasting 1-3 times with or without thermal cycling. Consistently, the results of failure type observed on specimens were not affected by recasting and thermal cycling in the 2 ceramic alloys. Although 3-point bending test was recommended by ISO 9693 for evaluating the metal-ceramic bond strength, some authors considered the shear test to be a more adequate and reliable method because there were less variables and residual stress at the metal-ceramic interface. [10-13] In addition, the results of the shear test are not influenced by Young's modulus of the alloy. [14] Moreover, Ucar et al. [15] found that the 3-point bending test and shear test shared a similar result in the metal-ceramic bond strength. Therefore, we chose to conduct shear test to determine the difference of bond strength among the alloys after recasting 1-3 times.

To explore the causes of these study results, there are two possible reasons. One is melting method in different studies. Ceramic alloys will likely change their properties after recasting resulting from evaporation, oxidation, or contamination in the process of melting. In this study, the melting method was arc melting with argon. It was a more favorable condition for recasting alloys because argon is hard to dissolve in molten liquid alloy or to react with the component of alloys. This will effectively protect the components of alloys from oxidation. In the study of Peraire et al. [16], after recasting high noble and noble alloys 7 times in a vacuum casting condition, the components of noble alloys and the major constituents of high noble ceramic alloy remained stable. The other possible reason is the treatment of previously melted ceramic alloys before recasting. Most of the contamination in previously cast alloys come from investment materials, oxides (such as $\mathrm{Al}_{2} \mathrm{O}_{3}, \mathrm{Fe}_{2} \mathrm{O}_{3}$ and $\mathrm{SiO}_{2}$ ), reaction layers, and airborneparticle abrasion with $\mathrm{Al}_{2} \mathrm{O}_{3}$. In this study, previously cast alloys were treated before recasting following the established protocols from our group, which successfully removed the contamination from recasting process and airborne-particle abrasion. [4] Compared to the new alloys after polishing, the impurity element was not detected in previously cast alloys after being treated. Hence, the combined effects of arc melting with argon and the treatment of ceramic alloys before recasting might be the underlying reasons for the results of our study.

The metal-ceramic bond strength would be strong or weak if the fracture type was cohesive or adhesive, respectively. Compared with the fracture mode with the bond strength, it seemed that there was no direct correlation between the type of failure and the bond strength. When it comes to the thickness of metal-ceramic reaction zone, we could find $\mathrm{Pd}-\mathrm{Cu}-\mathrm{Ga}$ alloy was higher than the Au-Pt alloy. Therefore, we can speculate that the thickness of metalceramic interfacial region might be the related factor of the failure mode, but it still needs to be further researched.

However, even without significant differences, there was still decline in the bond strength of each alloy after recasting 1-3 times based on the data in this study. The inevitable element loss in the melting process might be responsible for the slight decline of metal-ceramic bond strength. For example, in the $\mathrm{Pd}-\mathrm{Cu}-\mathrm{Ga}$ group, the peak of $\mathrm{Ga}$ in third recasting, which is the foundation of the metal-ceramic combination by bonding with $\mathrm{Cu}[17,18]$, was lower than that in first and second recastings. It also has been reported that after recasting 7 times in a vacuum-conditions, $\mathrm{Zn}$ and $\mathrm{Sn}$ in high noble alloy declined significantly by $50 \%$ and $100 \%$, respectively. Thus, it implied that the quality of metal porcelain bonding might be reduced if the number of recasting exceeds two or three.

Thermal cycling, which is commonly utilized to simulate oral long-term condition in vitro, can trigger repetitive stress on metal-ceramic interface, thus weakening the bond 
strength [19-21]. Moreover, discrepancy between the thermal expansion coefficients of the two combined materials during the process of thermal cycling can also affect adhesive strength. Hence, thermal cycling is considered a way of aging the ceramic-metal interface $[22,23]$. In addition, 3,000 thermal cycles were reported to be equivalent to 2.5 years of clinical application [24]. Based on these conditions, that means that metal-ceramic compatibility of 2 tested alloys was not significantly affected by recasting 1-3 times in shortmedium term aging. However, long-term aging effect should be further studied.

\section{Conclusions}

Within the limitations of this study, Pd-Cu-Ga and $\mathrm{Au}-\mathrm{Pt}$ ceramic alloys can be recycled 2 times without significant decrease on metal-ceramic interface properties, with or without thermal cycling. But giving thought to the labor and material costs of the treatment protocols with the alloys previously cast, it is more recommended that hulk alloys recycling is conducted by manufacturers.

\section{Data Availability}

The data used to support the findings of this study are available from the corresponding author upon request.

\section{Additional Points}

Clinical significance. After special treatment before recasting and under arc melting with argon, $\mathrm{Pd}-\mathrm{Cu}-\mathrm{Ga}$ and $\mathrm{Au}-\mathrm{Pt}$ alloys can be recycled 2 times without significant changes in the metal-ceramic interface.

\section{Conflicts of Interest}

The authors declare that they have no conflicts of interest.

\section{Authors' Contributions}

Yinghui Wang and Honglan Huang contributed equally to this work.

\section{Acknowledgments}

This work was partly supported by the National Nature Science Foundation of China (81641158), Nature Science Foundation of Fujian Province (2016J01354), Innovation Medical Program of Fujian Province (2015-CX-29), Colleges Special Foundation of Fujian Province Education Department (JAT160212), and Youth Foundation of Fujian Province Health Department (2014-2-24). The authors thank Mr. Weiqing $\mathrm{Wu}$, the Testing Center, Fuzhou University, Fujian, China and Mr. Jinming Wang, School of Material, Xiamen University, Fujian, China for technical support.

\section{References}

[1] I. Sailer, N. A. Makarov, D. S. Thoma, M. Zwahlen, and B. E. Pjetursson, "All-ceramic or metal-ceramic tooth-supported fixed dental prostheses (FDPs)? A systematic review of the survival and complication rates. Part I: Single crowns (SCs)," Dental Materials, vol. 31, no. 6, pp. 603-623, 2015.

[2] N. Naenni, A. Bindl, C. Sax, C. Hammerle, and I. Sailer, "A randomized controlled clinical trial of 3-unit posterior zirconia-ceramic fixed dental prostheses (FDP) with layered or pressed veneering ceramics: 3-year results," Journal of Dentistry, vol. 43, no. 11, pp. 1365-1370, 2015.

[3] A. S. Vaillant-Corroy, P. Corne, P. De March, S. Fleutot, and F. Cleymand, "Influence of recasting on the quality of dental alloys: a systematic review," The Journal of Prosthetic Dentistry, vol. 114, no. 2, pp. 205-211.e3, 2015.

[4] H. Lin, H. Zhang, X. Li, and H. Cheng, "Treatment of multiple ceramic alloys before recasting," The Journal of Prosthetic Dentistry, vol. 110, no. 1, pp. 29-40, 2013.

[5] S. Yang, H. Cheng, X. R. Li, W. Q. Wu, M. Zheng, and Y. H. Wang, "Study of the mechanical properties of Co-Cr ceramic alloy after recasts," West China Journal of Stomatology, vol. 29, no. 3, pp. 249-252, 2011.

[6] H. Cheng, S. Yang, X. Li, W. Wu, M. Zheng, and Y. Wang, "Physical properties of Au-Pt ceramic alloy after recasting," West China Journal of Stomatology, vol. 31, no. 4, pp. 412414, 2013.

[7] S. Yang, H. Cheng, X. R. Li, W. Q. Wu, M. Zheng, and Y. H. Wang, "Study of the mechanical properties of CP Ti ceramic alloy after recasts," Shanghai kou qiang yi xue = Shanghai Jour nal of Stomatology, vol. 23, no. 6, pp. 651-653, 2014.

[8] British Standard, Hardmetals-Metallographic determination of microstructure, Part 1: Photomicrographs and description, ISO, 2008.

[9] W. J. Garbelini, G. E. Henriques, M. Troia Junior, M. F. Mesquita, and C. C. Dezan, "Evaluation of low-fusing ceramic systems combined with titanium grades II and V by bending test and scanning electron microscopy," Journal of Applied Oral Science, vol. 11, no. 4, pp. 354-360, 2003.

[10] R. R. Wang and A. Fenton, "Titanium for prosthodontic applications: a review of the literature," Quintessence International, vol. 27, no. 6, pp. 401-408, 1996.

[11] I. A. Hammad, R. J. Goodkind, and W. W. Gerberich, "A shear test for the bond strength of ceramometals," The Journal of Prosthetic Dentistry, vol. 58, no. 4, pp. 431-437, 1987.

[12] Y. Taira, H. Matsumura, K. Yoshida, T. Tanaka, and M. Atsuta, "Influence of surface oxidation of titanium on adhesion," Journal of Dentistry, vol. 26, no. 1, pp. 69-73, 1998.

[13] E. Papazoglou, W. A. Brantley, W. M. Johnston, and A. B. Carr, "Effects of dental laboratory processing variables and in vitro testing medium on the porcelain adherence of highpalladium casting alloys," The Journal of Prosthetic Dentistry, vol. 79, no. 5, pp. 514-519, 1998.

[14] I. A. Hammad and Y. F. Talic, "Designs of bond strength tests for metal-ceramic complexes: review of the literature," The Journal of Prosthetic Dentistry, vol. 75, no. 6, pp. 602-608, 1996.

[15] Y. Ucar, Z. Aksahin, and C. Kurtoglu, "Metal ceramic bond after multiple castings of base metal alloy," The Journal of Prosthetic Dentistry, vol. 102, no. 3, pp. 165-171, 2009.

[16] M. Peraire, J. Martinez-Gomis, J. M. Anglada, J. Bizar, J. Salsench, and F. J. Gil, "Effects of recasting on the chemical composition, microstructure, microhardness, and ion release of 3 dental casting alloys and titanium," The International Journal of Prosthodontics, vol. 20, no. 3, pp. 286-288, 2007. 
[17] I. C. Pang, J. L. Gilbert, J. Chai, and E. P. Lautenschlager, "Bonding characteristics of low-fusing porcelain bonded to pure titanium and palladium-copper alloy," The Journal of Prosthetic Dentistry, vol. 73, no. 1, pp. 17-25, 1995.

[18] J. A. Hautaniemi, J. T. Juhanoja, E. J. Suoninen, and A. U. Yli-Urpo, "Oxidation of four palladium-rich ceramic fusing alloys,” Biomaterials, vol. 11, no. 1, pp. 62-72, 1990.

[19] M. S. Gale and B. W. Darvell, "Thermal cycling procedures for laboratory testing of dental restorations," Journal of Dentistry, vol. 27, no. 2, pp. 89-99, 1999.

[20] A. Leibrock, M. Degenhart, M. Behr, M. Rosentritt, and G. Handel, "In vitro study of the effect of thermo- and loadcycling on the bond strength of porcelain repair systems," Journal of Oral Rehabilitation, vol. 26, no. 2, pp. 130-137, 1999.

[21] M. G. Troia Jr., G. E. Henriques, M. A. Nobilo, and M. F. Mesquita, "The effect of thermal cycling on the bond strength of low-fusing porcelain to commercially pure titanium and titanium-aluminium-vanadium alloy," Dental Materials, vol. 19, no. 8, pp. 790-796, 2003.

[22] S. N. White, Z. C. Li, Z. Yu, and V. Kipnis, "Relationship between static chemical and cyclic mechanical fatigue in a feldspathic porcelain," Dental Materials, vol. 13, no. 2, pp. 103-110, 1997.

[23] R. Poljak-Guberina, A. Catovic, V. Jerolimov, M. Franz, and V. Bergman, "The fatigue strength of the interface between Ag-Pd alloy and hydrothermal ceramic," Dental Materials, vol. 15, no. 6, pp. 417-420, 1999.

[24] J. Fischer, C. Zbaren, B. Stawarczyk, and C. H. Hammerle, "The effect of thermal cycling on metal-ceramic bond strength," Journal of Dentistry, vol. 37, no. 7, pp. 549-553, 2009. 